\title{
Ergodic Capacity of Cognitive TAS/GSC Relaying in Nakagami-m Fading Channels
}

\author{
Yansha Deng*, Lifeng Wang*, Maged Elkashlan*, Kyeong Jin Kim ${ }^{\dagger}$, and Trung Q. Duong ${ }^{\ddagger}$ \\ *School of Electronic Engineering and Computer Science, Queen Mary University of London, London, UK \\ ${ }^{\dagger}$ Mitsubishi Electric Research Laboratories (MERL), 201 Broadway, Cambridge, MA, USA \\ ${ }_{\ddagger}$ School of Computing, Blekinge Institute of Technology, Karlskrona, Sweden
}

\begin{abstract}
We examine the impact of transmit antenna selection with receive generalized selection combining (TAS/GSC) for cognitive decode-and-forward (DF) relaying in Nakagami$m$ fading channels. We select a single transmit antenna at the secondary transmitter which maximizes the receive signal-tonoise ratio (SNR) and combine a subset of receive antennas with the largest SNRs at the secondary receiver. In an effort to assess the performance, we first derive the probability density function and cumulative distribution function of the end-to-end SNR using the moment generating function. We then derive new exact closed-form expression for the ergodic capacity. More importantly, by deriving the asymptotic expression for the high SNR approximation of the ergodic capacity, we gather deep insights into the high SNR slope and the power offset. Our results show that the high SNR slope is $\frac{1}{2}$ under the proportional interference power constraint. Under the fixed interference power constraint, the high SNR slope is zero.
\end{abstract}

\section{INTRODUCTION}

Recently, cognitive radio has rekindled enormous interest among academic and industrial communities due to its efficiency in spectrum utilization. In underlay spectrum sharing, the secondary users (SUs) are allowed to access the spectrum allocated to primary users (PUs) as long as the interference generated by the secondary transmission is restricted below a certain threshold [1]. Relaying, which is known as a costeffective approach for supporting high data rates in long distance applications, is well-suited for underlay spectrum sharing where the secondary network is power constrained. Hence, considerable attention has been attracted in this context. In [2], the impact of interference constraint on the outage probability of cognitive decode-and-forward (DF) relay was analyzed. In [3], the outage probability of cognitive relay network consisting of multiple primary transceivers and single relay was evaluated over Rayleigh fading. It has been shown that the interference power constraint gives rise to the outage saturation phenomenon $[2,3]$.

Multiple-input multiple-output (MIMO) opens up a new dimension for cognitive radio [4]. For example, as shown in [5], substantial capacity gains are achieved by employing MIMO. In [6], the effect of transmit antenna selection with receive maximal ratio combining (TAS/MRC) on the the ergodic capacity was analyzed. In [7], the effective capacity of spectrum sharing networks with generalized selection combining (GSC) at the SU was derived.

Different from the aforementioned works, in this paper, we view the cognitive relay network from the viewpoint of
TAS/GSC as an effective design for reliability enhancemen$\mathrm{t}$ and interference suppression. It has been acknowledged that TAS is an important component for the uplink of $4 \mathrm{G}$ long term evolution (LTE) and LTE Advanced because of its low feedback demand [8]. The GSC offers a performance/implementation tradeoff between maximal-ratio combining (MRC) and selection combining (SC).

Our objective in this paper is to examine the impact of TAS/GSC in underlay cognitive relay networks over Nakagami- $m$ fading. With this in mind, we derive new closedform expressions for the cumulative distribution function (CDF) and the probability density function (PDF) for the single hop signal-to-noise ratio (SNR) using the moment generating function (MGF) approach. Based on these new statistics, we first derive a new exact closed-form expression for the ergodic capacity of cognitive TAS/GSC in the presence of a DF relay in Nakagami- $m$ fading channels. We then derive tight high SNR approximations of the ergodic capacity for two cases: 1) proportional interference power constraint, and 2) fixed interference power constraint. Under the proportional interference power constraint, we confirm that the high SNR slope maintains the same regardless of $N_{S}, N_{R}$ and $N_{D}$. Under the fixed interference power constraint, we highlight that a capacity ceiling exists.

\section{System AND Channel Description}

We consider a dual-hop cognitive DF relay network consisting of the secondary source (S) with $N_{S}$ antennas, the secondary relay (R) with $N_{R}$ antennas, the secondary destination (D) with $N_{D}$ antennas, and single primary receiver (PU) with only one antenna. Both the primary channel and the secondary channel are assumed to undergo quasi-static fading with independent identically distributed (i.i.d.) Nakagami- $m$ distribution. Let $\left\{g_{1 i j}\right\}$ denote the channel coefficients of the $N_{\mathrm{S}} \times N_{\mathrm{R}}$ channels from the $\mathrm{S}$ to the $\mathrm{R}, i \in\left\{1, \ldots, N_{S}\right\}$, $j \in\left\{1, \ldots, N_{R}\right\},\left\{g_{2 j k}\right\}$ denote the channel coefficients of the $N_{\mathrm{R}} \times N_{\mathrm{D}}$ channels from the R to the $\mathrm{D}, k \in\left\{1, \ldots, N_{D}\right\}$, $h_{1 i}$ denote the channel coefficients of the $N_{S} \times 1$ channels from the $\mathrm{S}$ to the PU and $\left\{h_{2 j}\right\}$ denote the channel coefficients of the $N_{R} \times 1$ channels from the R to the PU. The channel coefficients follow Nakagami- $m$ distribution with fading parameters $m_{g 1}, m_{g 2}, m_{h 1}, m_{h 2}$, and average channel power gains $\Omega_{g 1}, \Omega_{g 2}, \Omega_{h 1}, \Omega_{h 2}$, respectively. In the following, $\|\cdot\|$ 
is the Euclidean norm, $|\cdot|$ is the absolute value, and $\mathbb{E}[\cdot]$ is the expectation.

Based on GSC, in the $\mathrm{S} \rightarrow \mathrm{R}$ link, we first sort $\left\{\left|g_{1 i j}\right|^{2}\right\}_{j=1}^{N_{R}}$ in descending order to obtain $\left|g_{1 i(1)}\right|^{2} \geq\left|g_{1 i(2)}\right|^{2} \geq \cdots \geq$ $\left|g_{1 i\left(N_{R}\right)}\right|^{2} \geq 0$. We then combine the first $L_{R}\left(1 \leq L_{R} \leq N_{R}\right)$ variable(s) to obtain $\theta_{i}=\sum_{j=1}^{L_{R}}\left|g_{1 i(j)}\right|^{2}$ at $\mathrm{R}$. The index of the selected transmit antenna at $\mathrm{S}$ is determined as $i^{*}=$ $\arg \max _{1 \leq i \leq N_{S}}\left\{\theta_{i}\right\}$. As such, the selected channel vector can be denoted as $\mathbf{g}_{1 i^{*} \theta_{i^{*}}}=\left[g_{1 i^{*}(1)}, \cdots, g_{1 i^{*}\left(L_{R}\right)}\right]$. The same antenna selection scheme is operated in the $\mathrm{R} \rightarrow \mathrm{D}$ link, we denote the selected channel vector as $\mathbf{g}_{2 j^{*} \theta_{j^{*}}}=$ $\left[g_{2 j^{*}(1)}, \cdots, g_{2 j^{*}\left(L_{D}\right)}\right]$ with $L_{D}\left(1 \leq L_{D} \leq N_{D}\right)$.

In the underlay cognitive relay network, the transmit powers at $\mathrm{S}$ and $\mathrm{R}$ are constrained according to

$$
P_{S}=\min \left(P, \frac{Q}{\left|h_{1 i^{*}}\right|^{2}}\right), P_{R}=\min \left(P, \frac{Q}{\left|h_{2 j^{*}}\right|^{2}}\right),
$$

where $P_{S}$ and $P_{R}$ are the maximum transmission powers at $\mathrm{S}$ and $\mathrm{R}$, and $Q$ is the maximum permissible interference power.

The instantaneous end-to-end SNR of the spectrum sharing network with TAS/GSC and DF relaying is defined as $\gamma=$ $\min \left\{\gamma_{1}, \gamma_{2}\right\}$, where the instantaneous SNR of the $\mathrm{S} \rightarrow \mathrm{R}$ link is

$$
\gamma_{1}=\min \left(\left\|\mathbf{g}_{1 i^{*} \theta_{i^{*}}}\right\|^{2} \bar{\gamma}_{P}, \frac{\left\|\mathbf{g}_{1 i^{*} \theta_{i^{*}}}\right\|^{2} \bar{\gamma}_{Q}}{\left|h_{1 i^{*}}\right|^{2}}\right)
$$

and the instantaneous SNR of the $\mathrm{R} \rightarrow \mathrm{D}$ link is

$$
\gamma_{2}=\min \left(\left\|\mathbf{g}_{2 j^{*} \theta^{*}}\right\|^{2} \bar{\gamma}_{P}, \frac{\left\|\mathbf{g}_{2 j^{*} \theta_{j^{*}}}\right\|^{2} \bar{\gamma}_{Q}}{\left|h_{2 j^{*}}\right|^{2}}\right) .
$$

In (2) and (3), we define $\bar{\gamma}_{P}=\frac{P}{N_{0}}$ and $\bar{\gamma}_{Q}=\frac{Q}{N_{0}}$, where $N_{0}$ is the noise power of an additive white Gaussian noise (AWGN).

\section{New Statistical Properties}

In this section, we first derive the $\mathrm{CDF}$ and PDF of $\left\|\mathbf{g}_{1 i^{*} \theta_{i^{*}}}\right\|^{2}$ as the following theorem.

Theorem 1: The CDF and PDF of $\left\|\mathbf{g}_{1 i^{*} \theta_{i^{*}}}\right\|^{2}$ are derived as

$$
\begin{aligned}
& F_{\left\|\mathbf{g}_{1 i^{*} \theta_{i *}}\right\|^{2}}(x)=\left(\frac{L_{R}}{\left(m_{g 1}-1\right) !}\left(\begin{array}{c}
N_{R} \\
L_{R}
\end{array}\right)\right)^{N_{S}} N_{S} !
\end{aligned}
$$

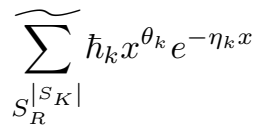

and

$$
f_{\left\|\mathbf{g}_{1 i^{*} \theta^{*}}\right\|^{2}}(x)=\frac{\left(\frac{L_{R}}{\left(m_{g 1}-1\right) !}\left(\begin{array}{c}
N_{R} \\
L_{R}
\end{array}\right)\right)^{N_{S}} N_{S} !}{\sum_{S_{R}^{\left|S_{K}\right|}} \hbar_{k} x^{\theta_{k}-1} e^{-\eta_{k} x}\left(\theta_{k}-\eta_{k} x\right),}
$$

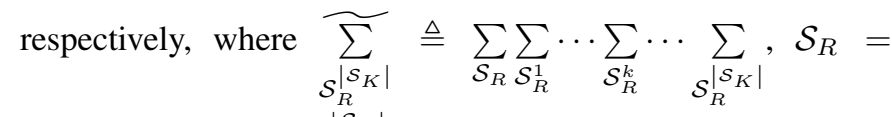
$\left\{\left(n_{\tau, 1}, \ldots, n_{\tau,\left|\mathcal{S}_{K}\right|}\right) \mid \sum_{k=1}^{\left|\mathcal{S}_{K}\right|} n_{\tau, k}=N_{S}\right\}$ with $\left\{n_{\tau, k}\right\} \in \mathbb{Z}^{+}$,
$\left|\mathcal{S}_{K}\right|$ is the cardinality of the set $\mathcal{S}_{K}$, and $\mathcal{S}_{K}$ denotes a set of $\left(2 m_{g 1}+1\right)$-tuples satisfying the following condition

$$
\begin{aligned}
\mathcal{S}_{K}= & \left\{\left(n_{k, 0}^{\Phi}, \ldots, n_{k, m_{g 1}-1}^{\Phi}, n_{k, 0}^{F}, \ldots, n_{k, m_{g 1}}^{F}\right) \mid\right. \\
& \left.\sum_{i=0}^{m_{g 1}-1} n_{k, i}^{\Phi}=L_{R}-1 ; \sum_{j=0}^{m_{g 1}} n_{k, j}^{F}=N_{R}-L_{R}\right\},
\end{aligned}
$$

thereby $\left|\mathcal{S}_{K}\right|=\left(\begin{array}{c}m_{g 1}+L_{R}-2 \\ m_{g 1}-1\end{array}\right)\left(\begin{array}{c}m_{g 1}+N_{R}-L_{R} \\ m_{g 1}\end{array}\right)$, and $\mathcal{S}_{R}^{k}=$ $\left\{\left(n_{\rho_{k}, 0}, \ldots, n_{\rho_{k}, m_{g 1} L_{R}+b_{k}^{F}}\right) \mid \sum_{n=0}^{m_{g 1} L_{R}+b_{k}^{F}} n_{\rho_{k}, n}=n_{\tau, k}\right\}, k=$ $1, \cdots,\left|\mathcal{S}_{K}\right|$, with $\left\{n_{k, i}^{\Phi}\right\},\left\{n_{k, i}^{\Phi}\right\},\left\{n_{k, j}^{F}\right\}$, and $\left\{n_{\rho_{k}, n}\right\} \in$ $\mathbb{Z}^{+}$. In (4) and (5), $\hbar_{k}, \theta_{k}$, and $\eta_{k}$ are respectively given by

$$
\begin{aligned}
\hbar_{k}= & \prod_{k=1}^{\left|\mathcal{S}_{K}\right|}\left(a_{k}^{\Phi} a_{k}^{F} \frac{\left(n_{2}-1\right) !}{L_{R}^{n_{2}}}\right)^{n_{\tau, k}}\left(\frac{\prod_{n=0}^{m_{g 1} L_{R}+b_{k}^{F}} \ell_{k}(n)^{n_{\rho_{k}, n}}}{\prod_{n=0}^{m_{k}+b_{k}^{F}} n_{\rho_{k}, n} !}\right), \\
\theta_{k}= & \sum_{k=1}^{\left|\mathcal{S}_{K}\right|} \sum_{n=0}^{m_{g 1} L_{R}+b_{k}^{F}} \mu_{k}(n) n_{\rho_{k}, n} \\
\eta_{k}= & \sum_{k=1}^{\left|\mathcal{S}_{K}\right|} \sum_{n=0}^{m_{g 1} L_{R}+b_{k}^{F}} \nu_{k}(n) n_{\rho_{k}, n}
\end{aligned}
$$

where $n_{2}=b_{k}^{\Phi}+b_{k}^{F}+m_{g 1}$. In (7) and (8), we have defined $\mu_{k}(n)$

$=\left\{\begin{array}{lc}0, & n=0 \\ n-1, & 1 \leq n \leq m_{g 1}\left(L_{R}-1\right)-b_{k}^{\Phi} \\ n-\operatorname{sgn}\left(c_{k}^{F}\right) & \left(m_{g 1}\left(L_{R}-1\right)-b_{k}^{\Phi}\right)-1 \\ & m_{g 1}\left(L_{R}-1\right)-b_{k}^{\Phi}<n \leq m_{g 1} L_{R}+b_{k}^{F}\end{array}\right.$

$$
\begin{aligned}
& \nu_{k}(n) \\
& = \begin{cases}0, & n=0 \\
\frac{m_{g 1}}{\Omega_{g 1}}, & 1 \leq n \leq m_{g 1}\left(L_{R}-1\right)-b_{k}^{\Phi} \\
\frac{c_{k}^{F}}{L_{R}}+\frac{m_{g 1}}{\Omega_{g 1}}, & m_{g 1}\left(L_{R}-1\right)-b_{k}^{\Phi}<n \leq m_{g 1} L_{R}+b_{k}^{F} .\end{cases}
\end{aligned}
$$

In (6), $a_{k}^{\Phi}, a_{k}^{F}, b_{k}^{\Phi}, b_{k}^{F}$ and $c_{k}^{F}$, and $\ell_{k}(n)$ are given by

$$
\begin{aligned}
& a_{k}^{\Phi}=\frac{\left(L_{R}-1\right) !}{\prod_{i=0}^{m_{g 1}-1} n_{k, i}^{\Phi} !} \prod_{i=0}^{m_{g 1}-1}\left(\frac{1}{i !}\right)^{n_{k, i}^{\Phi}}, b_{k}^{\Phi}=\sum_{i=0}^{m_{g 1}-1} n_{k, i}^{\Phi} i \\
& a_{k}^{F}=\frac{\left(N_{R}-L_{R}\right) !}{\prod_{j=0}^{m_{g 1}} n_{k, j}^{F} !} \prod_{j=0}^{m_{g 1}-1}\left(\frac{-1}{j !}\right)^{n_{k, j+1}^{F}}\left(\frac{m_{g 1}}{\Omega_{g 1}}\right)^{b_{k}^{F}}, \\
& b_{k}^{F}=\sum_{j=0}^{m_{g 1}-1} j n_{k, j+1}^{F}, c_{k}^{F}=\frac{m_{g 1}}{\Omega_{g 1}} \sum_{j=1}^{m_{g 1}} n_{k, j}^{F},
\end{aligned}
$$




$$
\begin{aligned}
& \ell_{k}(n) \\
& =\left\{\begin{array}{c}
\left(\frac{m_{g 1}}{\Omega_{g 1}}\right)^{\mu_{k}(n)-b_{k}^{F}}\left(\frac{1}{L_{R}} \sum_{j=1}^{m_{g 1}} n_{k, j}^{F}+1\right)^{-n_{2}} \\
n=0 \\
\left(\frac{m_{g 1}}{\Omega_{g 1}}\right)^{\mu_{k}(n)-b_{k}^{F}}\left(\Upsilon_{k 1}+\Upsilon_{k 2}-\frac{1-\operatorname{sgn}\left(c_{k}^{F}\right)}{(n-1) !}\right) \\
1 \leq n \leq m_{g 1}\left(L_{R}-1\right)-b_{k}^{\Phi} \\
\left(\frac{m_{g 1}}{\Omega_{g 1}}\right)^{\mu_{k}(n)-b_{k}^{F}}\left(\Upsilon_{k 3}+\Upsilon_{k 4}-\frac{1-\operatorname{sgn}\left(c_{k}^{F}\right)}{(n-1) !}\right) \\
m_{g 1}\left(L_{R}-1\right)-b_{k}^{\Phi}<n \leq m_{g 1} L_{R}+b_{k}^{F}
\end{array}\right.
\end{aligned}
$$

where

$$
\begin{aligned}
\Upsilon_{k 1}= & -\frac{\operatorname{sgn}\left(c_{k}^{F}\right)}{(n-1) !}\left(\frac{1}{L_{R}} \sum_{j=1}^{m_{g 1}} n_{k, j}^{F}+1\right)^{-n_{2}} \\
\Upsilon_{k 2}= & (-1)^{1-n_{1}} \frac{\operatorname{sgn}\left(c_{k}^{F}\right)}{(n-1) !} \sum_{l=1}^{n_{2}}\left(\frac{1}{L_{R}} \sum_{n=1}^{m_{g 1}} n_{k, n}^{F}+1\right)^{-\left(n_{2}-l+1\right)} \\
& \left(\begin{array}{c}
l-n_{1}-1 \\
l-1
\end{array}\right)\left(\frac{1}{L_{R}} \sum_{n=1}^{m_{g 1}} n_{k, n}^{F}\right)^{n_{1}-l} \\
\Upsilon_{k 3}= & -\frac{\operatorname{sgn}\left(c_{k}^{F}\right)}{\left(n_{1}-1\right) !}\left(\frac{1}{L_{R}} \sum_{j=1}^{m_{g 1}} n_{k, j}^{F}+1\right)^{-\left(n_{2}-n_{1}+1\right)} \\
\Upsilon_{k 4}= & \frac{\operatorname{sgn}\left(c_{k}^{F}\right)}{\left(n_{1}-1\right) !} \sum_{g 1}^{m_{g 1}}\left(L_{R}-1\right)-b_{k}^{\Phi} \\
& \left(\begin{array}{c}
n_{2}-n_{1}+l-1 \\
l-1
\end{array}\right)\left(\frac{1}{L_{R}} \sum_{j=1}^{m_{g 1}} n_{k, j}^{F}\right)^{-\left(n_{2}-n_{1}+l\right)}
\end{aligned}
$$

In (16), (17), and (18), $n_{1}=n-m_{g 1}\left(L_{R}-1\right)+b_{k}^{\Phi}$. Note that the proof is omitted due to space limit.

The CDF and PDF of $\left\|\mathbf{g}_{2 j^{*} \theta_{j^{*}}}\right\|^{2}$ follow from (4) and (5) by interchanging the parameters $m_{g 1} \rightarrow m_{g 2}, m_{h 1} \rightarrow m_{h 2}$, $\Omega_{h 1} \rightarrow \Omega_{h 2}, L_{R} \rightarrow L_{D}, N_{R} \rightarrow N_{D}, N_{S} \rightarrow N_{R}, \mathcal{S}_{R} \rightarrow$ $\mathcal{S}_{D},\left|\mathcal{S}_{K}\right| \rightarrow\left|\mathcal{S}_{T}\right|, \mathcal{S}_{R}^{\left|\mathcal{S}_{K}\right|} \rightarrow \mathcal{S}_{D}^{\left|\mathcal{S}_{T}\right|}, n_{\tau, k} \rightarrow n_{\tau, t}, n_{k, i}^{\Phi} \rightarrow$ $n_{t, i}^{\Phi}, n_{k, i}^{\Phi} \rightarrow n_{t, i}^{\Phi}, n_{k, j}^{F} \rightarrow n_{t, j}^{F}$, and $n_{\rho_{k}, n} \rightarrow n_{\rho_{t}, n}, \eta_{k} \rightarrow$

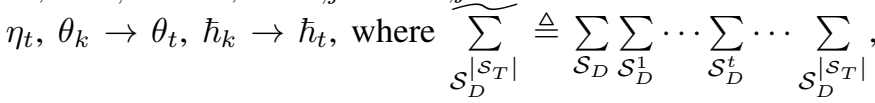
$\mathcal{S}_{D}=\left\{\left(n_{\tau, 1}, \ldots, n_{\tau,\left|\mathcal{S}_{T}\right|}\right) \mid \sum_{t=1}^{\left|\mathcal{S}_{T}\right|} n_{\tau, t}=N_{R}\right\}$ with $\left\{n_{\tau, t}\right\} \in$ $\mathbb{Z}^{+},\left|\mathcal{S}_{T}\right|$ is the cardinality of the set $\mathcal{S}_{T}$, and $\mathcal{S}_{T}$ denotes a set of $\left(2 m_{g 2}+1\right)$-tuples satisfying the following condition

$$
\begin{aligned}
\mathcal{S}_{T}= & \left\{\left(n_{t, 0}^{\Phi}, \ldots, n_{t, m_{g 2}-1}^{\Phi}, n_{t, 0}^{F}, \ldots, n_{t, m_{g 2}}^{F}\right) \mid\right. \\
& \left.\sum_{i=0}^{m_{g 2}-1} n_{t, i}^{\Phi}=L_{D}-1 ; \sum_{j=0}^{m_{g 2}} n_{t, j}^{F}=N_{D}-L_{D}\right\},
\end{aligned}
$$

thereby $\left|\mathcal{S}_{T}\right|=\left(\begin{array}{c}m_{g^{2}}+L_{D}-2 \\ m_{g 2}-1\end{array}\right)\left(\begin{array}{c}m_{g 2}+N_{D}-L_{D} \\ m_{g 2}\end{array}\right)$, and $\mathcal{S}_{D}^{t}=$ $\left\{\left(n_{\rho_{t}, 0}, \ldots, n_{\rho_{t}, m_{g 2} L_{D}+b_{t}^{F}}\right) \mid \sum_{n=0}^{m_{g 2} L_{D}+b_{t}^{F}} n_{\rho_{t}, n}=n_{\tau, t}\right\}, t=$ $1, \cdots,\left|\mathcal{S}_{T}\right|$, with $\left\{n_{t, i}^{\Phi}\right\},\left\{n_{t, i}^{\Phi}\right\},\left\{n_{t, j}^{F}\right\}$, and $\left\{n_{\rho_{t}, n}\right\} \in \mathbb{Z}^{+}$.
Based on (2), the CDF of $\gamma_{1}$ can be derived using the CDFs and PDFs of $\left\|\mathbf{g}_{1 i^{*} \theta_{i^{*}}}\right\|^{2}$ and $\left|h_{1 i^{*}}\right|^{2}$, the closed-form CDF of $\gamma_{1}$ is evaluated in the following theorem.

Theorem 2: The CDF of $\gamma_{1}$ is represented as

$$
F_{\gamma_{1}}(x)=\left(\frac{L_{R}}{\left(m_{g 1}-1\right) !}\left(\begin{array}{c}
N_{R} \\
L_{R}
\end{array}\right)\right)^{N_{S}} N_{S} ! \widetilde{\sum_{S_{R}^{\left|S_{K}\right|}}} \hbar_{k} \Xi_{k}(x),
$$

where

$$
\begin{aligned}
& \Xi_{k}(x)=\left(1-\frac{\Gamma\left(m_{h 1}, \frac{Q}{P} \frac{m_{h 1}}{\Omega_{h 1}}\right)}{\Gamma\left(m_{h 1}\right)}\right)\left(\frac{x}{\bar{\gamma}_{P}}\right)^{\theta_{k}} e^{-\eta_{k} \frac{x}{\bar{\gamma}_{P}}}+ \\
&\left(\frac{m_{h 1}}{\Omega_{h 1}}\right)^{m_{h 1}}\left(\frac{x}{\bar{\gamma}_{Q}}\right)^{\theta_{k}} \frac{\Gamma\left(\theta_{k}+m_{h 1},\left(\frac{m_{h 1}}{\Omega_{h 1}}+\frac{\eta_{k} x}{\bar{\gamma}_{Q}}\right) \frac{Q}{P}\right)}{\left(m_{h 1}-1\right) !\left(\frac{m_{h 1}}{\Omega_{h 1}}+\frac{\eta_{k} x}{\bar{\gamma}_{Q}}\right)^{\theta_{k}+m_{h 1}}} .
\end{aligned}
$$

Note that the proof is omitted due to space limit.

According to Theorem 2, the CDF of $\gamma_{2}$ is given by

$$
F_{\gamma_{2}}(x)=\left(\frac{L_{D}}{\left(m_{g 2}-1\right) !}\left(\begin{array}{c}
N_{D} \\
L_{D}
\end{array}\right)\right)^{N_{R}} N_{R} ! \widetilde{\sum_{S_{D}^{\left|S_{T}\right|}}} \hbar_{t} \Xi_{t}(x),
$$

where $\Xi_{t}(x)$ follows from $\Xi_{k}(x)$ by interchanging the parameters $m_{g 1} \rightarrow m_{g 2}, m_{h 1} \rightarrow m_{h 2}, \Omega_{h 1} \rightarrow \Omega_{h 2}, \eta_{k} \rightarrow \eta_{t}$, $\theta_{k} \rightarrow \theta_{t}$. Our new closed form expressions for CDFs of $\gamma_{1}$ and $\gamma_{2}$ are composed of easy-to-evaluate finite summations functions, such as the standard incomplete gamma function [9, eq.8.350.2], and are valid for arbitrary fading severity parameters of all links.

\section{ERGodic CAPACITY}

The ergodic capacity, also known as Shannon capacity, is an important performance indicator for cognitive underlay spectrum sharing. It is defined as the maximum achievable long-term rate, where no delay limit is taken into account. The ergodic capacity is expressed as [10]

$$
C_{\text {erg }}=\frac{1}{2 \ln 2} \int_{0}^{\infty} \frac{1-F_{\gamma}(x)}{1+x} d x .
$$

To simplify the analysis of (22), let us define $F_{\gamma_{1}}(x)=1+$ $\tilde{F}_{\gamma_{1}}(x)$ and $F_{\gamma_{2}}(x)=1+\tilde{F}_{\gamma_{2}}(x)$, therefore we rewrite (22) as

$$
C_{e r g}=\frac{1}{2 \ln 2} \int_{0}^{\infty} \frac{\tilde{F}_{\gamma_{1}}(x) \tilde{F}_{\gamma_{2}}(x)}{1+x} d x
$$

where

$$
\tilde{F}_{\gamma_{1}}(x)=\left(\frac{L_{R}}{\left(m_{g 1}-1\right) !}\left(\begin{array}{c}
N_{R} \\
L_{R}
\end{array}\right)\right)^{N_{S}} N_{S} ! \widetilde{\sum_{S_{R}^{\left|S_{K}\right|}}} \hbar_{k} \operatorname{sgn}\left(\eta_{k}\right) \Xi_{k}(x)
$$

and

$$
\tilde{F}_{\gamma_{2}}(x)=\left(\frac{L_{D}}{\left(m_{g 2}-1\right) !}\left(\begin{array}{c}
N_{D} \\
L_{D}
\end{array}\right)\right)^{N_{R}} N_{R} ! \widetilde{\sum_{S_{D}^{\left|S_{T}\right|}}} \hbar_{t} \operatorname{sgn}\left(\eta_{t}\right) \Xi_{t}(x) .
$$


For simplicity, we assume $m_{h 1}=m_{h 2}=m_{h}$ and $\Omega_{h 1}=$ $\Omega_{h 2}=\Omega_{h}$ in the following analysis. Substituting (24) and (25) into (23), we obtain a closed-form expression for the ergodic capacity.

A. Exact Analysis

Our new closed-form expression for the ergodic capacity of cognitive TAS/GSC relaying in Nakagami- $m$ fading channels is given in (26) at the top of next page. In (26),

$$
\begin{aligned}
& \nabla(\theta) \triangleq\left(1-\Gamma\left(m_{h}, \frac{Q}{P} \frac{m_{h}}{\Omega_{h}}\right) / \Gamma\left(m_{h}\right)\right)\left(\frac{1}{\bar{\gamma}_{P}}\right)^{\theta}, \\
& \Delta(\theta, \eta, j, k) \triangleq \frac{\left(\theta+m_{h}-1\right) !}{\left(m_{h}-1\right) !}\left(\frac{1}{\bar{\gamma}_{Q}}\right)^{\theta} e^{-\frac{m_{h} Q}{\Omega_{h} P}} \sum_{j=0}^{\theta+m_{h}-1} \frac{1}{j !} \\
& \left(\frac{Q}{P}\right)^{j} \sum_{k=0}^{j}\left(\begin{array}{l}
j \\
k
\end{array}\right)\left(\frac{m_{h}}{\Omega_{h}}\right)^{m_{h}+j-k}\left(\frac{\eta}{\bar{\gamma}_{Q}}\right)^{k}, \\
& \nu\left(\eta, l, k_{1}, k_{2}\right) \triangleq \Gamma(\tau)\left(\bar{\gamma}_{Q} m_{h} / \eta \Omega_{h}\right)^{\tau-l} \Psi(\tau, \tau+1-l ; \\
& \left.\left(\eta_{t}+\eta_{k}\right) \bar{\gamma}_{Q} m_{h} / \bar{\gamma}_{p} \eta \Omega_{h}\right), \\
& \partial(\eta, l) \triangleq \frac{(29)}{\left(\bar{\gamma}_{Q} m_{h} / \eta_{t} \Omega_{h}-1\right)^{\theta_{t}+m_{h}}\left(\bar{\gamma}_{Q} m_{h} / \eta_{k} \Omega_{h}-1\right)^{\theta_{k}+m_{h}}}, \\
& \kappa(\theta, \eta, l, j) \triangleq \frac{(-1)^{\theta+m_{h}-l+1}\left(j+\theta+m_{h}-l-1\right)}{\left(\bar{\gamma}_{Q} m_{h} / \Omega_{h}\right)^{j+\theta+m_{h}-l}\left(1 / \eta_{t}-1 / \eta_{k}\right)^{j+\theta+m_{h}-l}} .
\end{aligned}
$$

In (29), $\tau=\theta_{k}+k_{1}+\theta_{t}+k_{2}+1$.

The derived closed-form expression for the ergodic capacity consists of easy-to-compute finite summations functions, such as the standard incomplete gamma function [9, eq.8.350.2] and the confluent hypergeometric function $\Psi(\alpha, \gamma ; z)$ [9, eq.9.211.4.8]. Our result can be applied and simplified to the special cases of TAS/MRC and TAS/SC in Nakagami$m$ fading channel, as well as TAS/GSC in Rayleigh fading channels.

\section{B. High SNR Capacity analysis}

In order to examine the capacity performance in the high SNR regime with $\bar{\gamma}_{P} \rightarrow \infty$, we further derive the tight high SNR approximation of the ergodic capacity in closedform. With the aid of the Jensen's inequality, the high SNR approximation of the ergodic capacity is presented as $[11,12]$

$$
C_{\text {erg }}^{\infty}=\frac{1}{2} \mathbb{E}\left[\log _{2}(1+\gamma)\right] \leq \frac{1}{2} \log _{2} \mathbb{E}(1+\gamma) \approx \frac{1}{2} \log _{2} \mathbb{E}(\gamma),
$$

Therefore, we can rewrite (32) as

$$
C_{\text {erg }}^{\infty} \approx \frac{1}{2} \log _{2} \int_{0}^{\infty} \tilde{F}_{\gamma_{1}}(x) \tilde{F}_{\gamma_{2}}(x) d x .
$$

By plugging (24) and (25) into (33), the approximations of high SNR ergodic capacity with the proportional interference power constraint and the fixed interference power constraint are derived in the following content, respectively. Considering the page limitation, we omit the detailed derivations.
1) Proportional Interference Power Constraint:

When $Q$ is proportional to $P$, the high SNR approximation of the ergodic capacity is derived as

$$
C_{e r g}^{\infty} \approx \frac{1}{2} \log _{2}\left(\bar{\gamma}_{P}\right)+\frac{1}{2} \log _{2}(\Upsilon),
$$

where

$$
\begin{aligned}
& \Upsilon=\left(\frac{L_{R}}{\left(m_{g 1}-1\right) !}\left(\begin{array}{c}
N_{R} \\
L_{R}
\end{array}\right)\right)^{N_{S}} N_{S} ! \widetilde{\sum_{S_{R}^{\left|S_{K}\right|}}} \hbar_{k} \operatorname{sgn}\left(\eta_{k}\right) \\
& \left(\frac{L_{D}}{\left(m_{g 2}-1\right) !}\left(\begin{array}{c}
N_{D} \\
L_{D}
\end{array}\right)\right)^{N_{R}} N_{R} ! \widetilde{\sum_{S_{D}^{\left|S_{T}\right|}}} \hbar_{t} \operatorname{sgn}\left(\eta_{t}\right) \\
& {\left[\lambda^{2} \frac{\Gamma\left(\theta_{k}+\theta_{t}+1\right)}{\left(\eta_{k}+\eta_{t}\right)^{\theta_{k}+\theta_{t}+1}}+\lambda \sum_{j_{2}=0}^{\theta_{t}+m_{h}-1} \sum_{k_{2}=0}^{j_{2}} \Delta_{s}\left(\theta_{t}, j_{2}, k_{2}\right)\right.} \\
& \frac{\nu_{s}\left(\eta_{t}, \theta_{t}, 0, k_{2}\right)}{(P / Q)^{\theta_{k}+j_{2}+1}}+\lambda \sum_{j_{1}=0}^{\theta_{k}+m_{h}-1} \sum_{k_{1}=0}^{j_{1}} \frac{\Delta_{s}\left(\theta_{k}, j_{1}, k_{1}\right)}{(P / Q)^{\theta_{t}+j_{1}+1}} \\
& \nu_{s}\left(\eta_{k}, \theta_{k}, k_{1}, 0\right)+\sum_{j_{1}=0}^{\theta_{k}+m_{h}-1} \sum_{k_{1}=0}^{j_{1}} \sum_{j_{2}=0}^{\theta_{t}+m_{h}-1} \sum_{k_{2}=0}^{j_{2}} \Omega \\
& \left.\frac{\Delta_{s}\left(\theta_{k}, j_{1}, k_{1}\right) \Delta_{s}\left(\theta_{t}, j_{2}, k_{2}\right)}{\left(m_{h} / \Omega_{h}\right)^{\theta_{k}+\theta_{t}+1}(P / Q)^{j_{1}+j_{2}+1}}\right] \text {. }
\end{aligned}
$$

In (35), we have defined

$\Delta_{s}(\theta, j, k) \triangleq \frac{\left(\theta+m_{h}-1\right) !}{\left(m_{h}-1\right) ! j !} e^{-\frac{m_{h} Q}{\Omega_{h} P}}\left(\begin{array}{l}j \\ k\end{array}\right)\left(\frac{m_{h}}{\Omega_{h}}\right)^{\theta_{k}+\theta_{t}+j+1-\theta}$,

$\kappa_{s}(\theta, \eta, l) \triangleq \frac{(-1)^{\left(\theta+m_{h}-l\right)}\left(\begin{array}{c}\theta_{k}+\theta_{t}+2 m_{h}-l-1 \\ \theta+m_{h}-l\end{array}\right)}{\left(1 / \eta_{t}-1 / \eta_{k}\right)^{\theta_{k}+\theta_{t}+2 m_{h}-l} \eta^{k_{1}+k_{2}-l}}$,

$\nu_{s}\left(\eta, \varepsilon, k_{1}, k_{2}\right) \triangleq \Gamma\left(\theta_{k}+k_{1}+\theta_{t}+k_{2}+1\right)(1 / \eta)^{\theta_{k}+\theta_{t}+1}$

$\Psi\left(\theta_{k}+k_{1}+\theta_{t}+k_{2}+1, \theta_{k}+k_{1}+\theta_{t}+k_{2}+2-m_{h}-\varepsilon ;\right.$

$\left.\frac{\left(\eta_{t}+\eta_{k}\right) m_{h} Q}{\Omega_{h} \eta P}\right)$,

and

$$
\begin{aligned}
& \Omega \triangleq\left(1-\operatorname{sgn}\left(\left|\eta_{k}-\eta_{t}\right|\right)\right) \nu_{s}\left(\eta_{k}, \theta_{k}+\theta_{t}+m_{h}, k_{1}, k_{2}\right),
\end{aligned}
$$

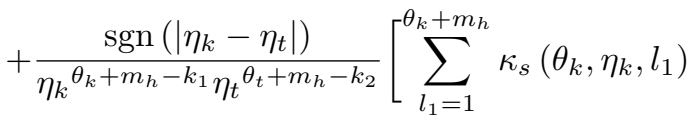

$$
\begin{aligned}
& \nu_{s}\left(\eta_{k}, l_{1}-m_{h}, k_{1}, k_{2}\right)+\sum_{l_{2}=1}^{\theta_{t}+m_{h}}(-1)^{\theta_{k}+\theta_{t}+2 m_{h}-l_{2}} \\
& \left.\kappa_{s}\left(\theta_{t}, \eta_{t}, l_{2}\right) \nu_{s}\left(\eta_{t}, l_{2}-m_{h}, k_{1}, k_{2}\right)\right] \text {. }
\end{aligned}
$$

Based on (34), we characterize two important parameters determining the affine approximation of the ergodic capacity in the high SNR regime, namely the high SNR slope and the high SNR power offset [13]. The high SNR slope is also known as degrees of freedom or multiplexing gain [14]. The high SNR power offset captures the delicate effect of different fading models, the number of antennas at each terminal, and 


$$
\begin{aligned}
& C_{\text {erg }}=\frac{1}{2 \ln 2}\left(\frac{L_{R}}{\left(m_{g 1}-1\right) !}\left(\begin{array}{c}
N_{R} \\
L_{R}
\end{array}\right)\right)^{N_{S}} N_{S} ! \widetilde{\sum_{\mathcal{S}_{R}^{\left|\mathcal{S}_{K}\right|}}} \hbar_{k} \operatorname{sgn}\left(\eta_{k}\right)\left(\frac{L_{D}}{\left(m_{g 2}-1\right) !}\left(\begin{array}{c}
N_{D} \\
L_{D}
\end{array}\right)\right)^{N_{R}} N_{R} ! \widetilde{\sum_{\mathcal{S}_{D} \mathcal{S}_{T} \mid}} \hbar_{t} \operatorname{sgn}\left(\eta_{t}\right)\left[\nabla\left(\theta_{k}\right)\right. \\
& \nabla\left(\theta_{t}\right) \nu\left(\bar{\gamma}_{Q} m_{h} / \Omega_{h}, 1,0,0\right)+\Delta\left(\theta_{t}, \eta_{t}, j_{2}, k_{2}\right) \nabla\left(\theta_{k}\right) \frac{\left(\nu\left(\bar{\gamma}_{Q} m_{h} / \Omega_{h}, 1,0, k_{2}\right)-\sum_{l_{2}=1}^{\theta_{t}+m_{h}} \frac{\nu\left(\eta_{t}, l_{2}, 0, k_{2}\right)}{\left(\bar{\gamma}_{Q} m_{h} / \eta_{t} \Omega_{h}-1\right)^{1-l_{2}}}\right)}{\left(m_{h} / \Omega_{h}-\eta_{t} / \bar{\gamma}_{Q}\right)^{\theta_{t}+m_{h}}} \\
& +\frac{\left(\nu\left(\bar{\gamma}_{Q} m_{h} / \Omega_{h}, 1, k_{1}, 0\right)-\sum_{l_{1}=1}^{\theta_{k}+m_{h}} \frac{\nu\left(\eta_{k}, l_{1}, k_{1}, 0\right)}{\left(\bar{\gamma}_{Q} m_{h} / \eta_{k} \Omega_{h}-1\right)^{1-l_{1}}}\right)}{\left(m_{h} / \Omega_{h}-\eta_{k} / \bar{\gamma}_{Q}\right)^{\theta_{k}+m_{h}}} \nabla\left(\theta_{t}\right) \Delta\left(\theta_{k}, \eta_{k}, j_{1}, k_{1}\right)+\Delta\left(\theta_{k}, \eta_{k}, j_{1}, k_{1}\right) \\
& \Delta\left(\theta_{t}, \eta_{t}, j_{2}, k_{2}\right)\left(\frac{\bar{\gamma}_{Q}}{\eta_{k}}\right)^{\theta_{k}+m_{h}}\left(\frac{\bar{\gamma}_{Q}}{\eta_{t}}\right)^{\theta_{t}+m_{h}}\left[\partial(\eta, 1) \nu\left(\bar{\gamma}_{Q} m_{h} / \Omega_{h}, 1, k_{1}, k_{2}\right)+\sum_{l_{1}=1}^{\theta_{k}+m_{h}}\left(-\partial\left(\eta_{k}, l_{1}\right)+\right.\right. \\
& \left.\sum_{j=1}^{\theta_{t}+m_{h}} \frac{\kappa\left(\theta_{k}, \eta_{k}, l_{1}, j\right)}{\left(\bar{\gamma}_{Q} m_{h} / \eta_{t} \Omega_{h}-1\right)^{\theta_{t}+m_{h}-j+1}}\right) \nu\left(\eta_{k}, l_{1}, k_{1}, k_{2}\right)+\operatorname{sgn}\left(\left|\eta_{k}-\eta_{t}\right|\right) \nu\left(\eta_{t}, l_{2}, k_{1}, k_{2}\right) \sum_{l_{2}=1}^{\theta_{t}+m_{h}}\left(-\partial\left(\eta_{t}, l_{2}\right)\right. \\
& \left.\left.\left.+\sum_{i=1}^{\theta_{k}+m_{h}} \frac{(-1)^{i+\theta_{t}+m_{h}-l_{2}}}{\left(\bar{\gamma}_{Q} m_{h} / \eta_{k} \Omega_{h}-1\right)^{\theta_{k}+m_{h}-i+1}} \kappa\left(\theta_{t}, \eta_{t}, l_{2}, i\right)\right)-\sum_{l_{3}=\theta_{k}+m_{h}+1}^{\theta_{k}+\theta_{t}+2 m_{h}} \frac{\left(1-\operatorname{sgn}\left(\left|\eta_{k}-\eta_{t}\right|\right)\right) \nu\left(\eta_{k}, l_{3}, k_{1}, k_{2}\right)}{\left(\bar{\gamma}_{Q} m_{h} / \eta_{k} \Omega_{h}-1\right)^{\theta_{k}+\theta_{t}+2 m_{h}-m+1}}\right]\right] .
\end{aligned}
$$

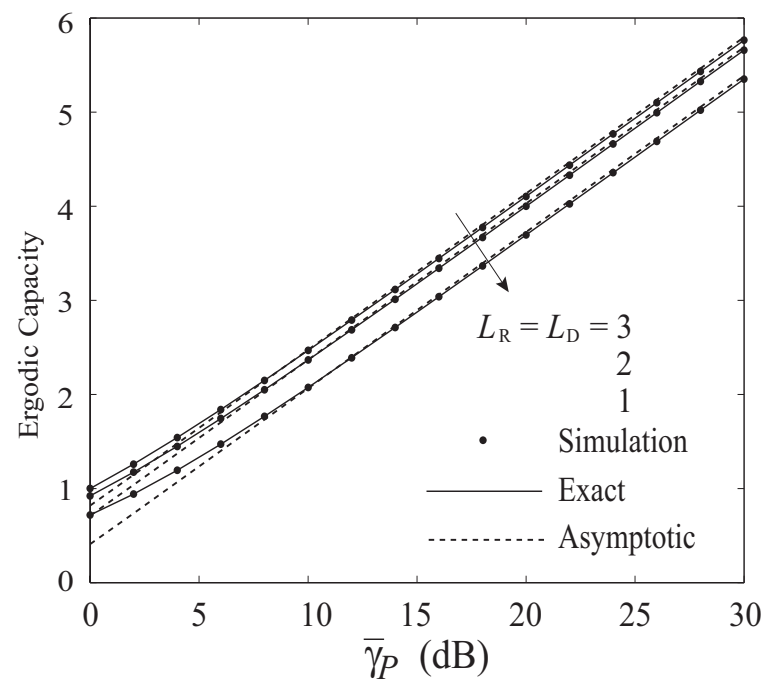

Fig. 1. Cognitive spectrum sharing with TAS/GSC and DF relaying: $N_{\mathrm{S}}=2$, $N_{\mathrm{R}}=3, N_{\mathrm{D}}=3, m_{g 1}=1, m_{g 2}=2, m_{h}=2, \Omega_{h}=1$, and $\bar{\gamma}_{Q}=2 \bar{\gamma}_{P}$.

the interference power constraint. We represent the high SNR approximation for the ergodic capacity as [13]

$$
C_{e r g}^{\infty} \approx S_{\infty}\left(\log _{2}\left(\bar{\gamma}_{P}\right)-\mathcal{L}_{\infty}\right),
$$

where $S_{\infty}$ is the high SNR slope in bits/s/Hz/(3 dB),

$$
S_{\infty}=\lim _{\bar{\gamma}_{P} \rightarrow \infty} \frac{C_{e r g}^{\infty}}{\log _{2}\left(\bar{\gamma}_{P}\right)}=\frac{1}{2},
$$

and $\mathcal{L}_{\infty}$ is the high SNR power offset in $3 \mathrm{~dB}$ units,

$$
\mathcal{L}_{\infty}=\lim _{\bar{\gamma}_{P} \rightarrow \infty}\left(\log _{2}\left(\bar{\gamma}_{P}\right)-\frac{C_{\text {erg }}^{\infty}}{S_{\infty}}\right)=\log _{2}(\Upsilon) .
$$

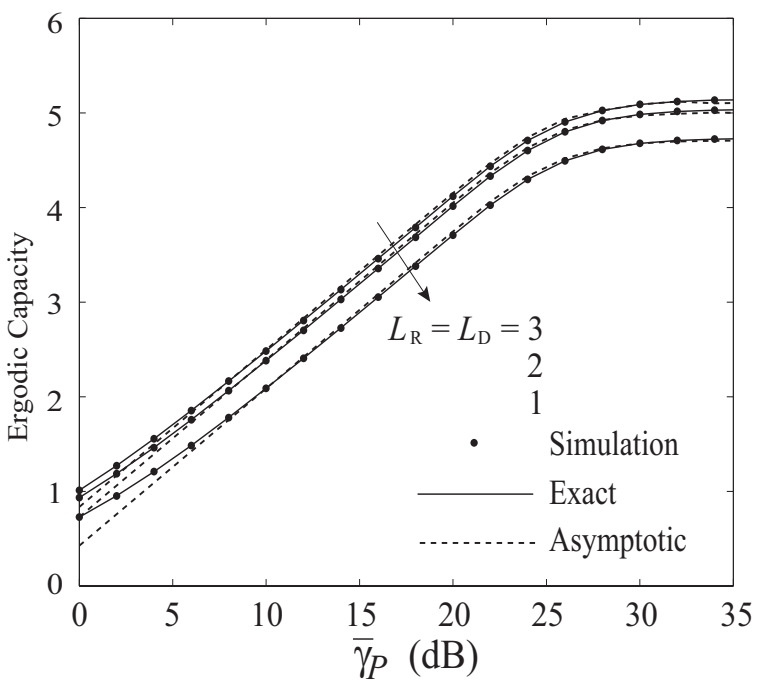

Fig. 2. Cognitive spectrum sharing with TAS/GSC and DF relaying: $N_{\mathrm{S}}=2$, $N_{\mathrm{R}}=3, N_{\mathrm{D}}=3, m_{g 1}=1, m_{g 2}=2, m_{h}=2, \Omega_{h}=1$, and $\bar{\gamma}_{Q}=25 \mathrm{~dB}$.

From (40), we see that the capacity slope $S_{\infty}$ is independent of the interference power constraint, the number of antennas at the source, relay, and destination, as well as the primary network. The high SNR power offset $\mathcal{L}_{\infty}$ is independent of $\bar{\gamma}_{P}$.

\section{2) Fixed Interference Power Constraint:}

When $Q$ is fixed, the high SNR approximation of the ergodic capacity is given in (43) at the top of the next page. From (43), we find that for the fixed interference power constraint, the high SNR slope collapses to zero. 


$$
\begin{aligned}
& C_{\text {erg }}^{\infty}=\frac{1}{2} \log _{2}\left[( \frac { L _ { R } } { ( m _ { g 1 } - 1 ) ! } ( \begin{array} { c } 
{ N _ { R } } \\
{ L _ { R } }
\end{array} ) ) ^ { N _ { S } } N _ { S } ! \widetilde { \sum _ { S _ { R } ^ { | S _ { K } | } } } \hbar _ { k } \operatorname { s g n } ( \eta _ { k } ) ( \frac { L _ { D } } { ( m _ { g 2 } - 1 ) ! } ( \begin{array} { c } 
{ N _ { D } } \\
{ L _ { D } }
\end{array} ) ) ^ { N _ { R } } N _ { R } ! \widetilde { \sum _ { S _ { D } ^ { | S _ { T } | } } } \hbar _ { t } \operatorname { s g n } ( \eta _ { t } ) \left[\lambda^{2} \bar{\gamma}_{P}\right.\right. \\
& \frac{\Gamma\left(\theta_{k}+\theta_{t}+1\right)}{\left(\eta_{k}+\eta_{t}\right)^{\theta_{k}+\theta_{t}+1}}+\bar{\gamma}_{Q}\left[\lambda \sum_{j_{2}=0}^{\theta_{t}+m_{h}-1} \sum_{k_{2}=0}^{j_{2}} \Delta_{s}\left(\theta_{t}, j_{2}, k_{2}\right) \nu_{s}\left(\eta_{t}, \theta_{t}, 0, k_{2}\right)\left(\frac{\bar{\gamma}_{Q}}{\bar{\gamma}_{P}}\right)^{\theta_{k}+j_{2}}+\lambda \sum_{j_{1}=0}^{\theta_{k}+m_{h}-1} \sum_{k_{1}=0}^{j_{1}}\left(\frac{\bar{\gamma}_{Q}}{\bar{\gamma}_{P}}\right)^{\theta_{t}+j_{1}}\right. \\
& \left.\left.\Delta_{s}\left(\theta_{k}, j_{1}, k_{1}\right) \nu_{s}\left(\eta_{k}, \theta_{k}, k_{1}, 0\right)+\sum_{j_{1}=0}^{\theta_{k}+m_{h}-1} \sum_{k_{1}=0}^{j_{1}} \sum_{j_{2}=0}^{\theta_{t}+m_{h}-1} \sum_{k_{2}=0}^{j_{2}} \frac{\Delta_{s}\left(\theta_{k}, j_{1}, k_{1}\right) \Delta_{s}\left(\theta_{t}, j_{2}, k_{2}\right)}{\left(m_{h} / \Omega_{h}\right)^{\theta_{k}+\theta_{t}+1}(P / Q)^{j_{1}+j_{2}}} \Omega\right]\right]
\end{aligned}
$$

\section{Numerical Results}

In this section, we present numerical results of the ergodic capacity for the cognitive TAS/GSC relaying in Nakagami- $m$ fading channels. We plot the ergodic capacity versus $\bar{\gamma}_{P}$ for different $L_{R}$ and $L_{D}$. All the figures clearly show that the exact curves are in precise agreement with the Monte Carlo simulations.

In Fig. 1, we plot the exact ergodic capacity and its high SNR approximation with proportional interference constraint from (26) and (34), respectively. We see that the high SNR approximations of the ergodic capacity are tight, which well predicts the behavior of the ergodic capacity in the high SNR regime. It is obvious that the ergodic capacity can be improved by increasing $L_{R}$ and $L_{D}$. The parallel curves prove that the high SNR slope is independent of $L_{R}$ and $L_{D}$.

In Fig. 2, we plot the impact of fixed interference constraint on the ergodic capacity. The exact ergodic capacity and its high SNR approximation are plotted from (26) and (43). Interestingly, we find that the capacity ceiling occurs beyond $30 \mathrm{~dB}$. This is due to the fact that when $\gamma_{P} \rightarrow \infty, \min \left(P, Q /\left|h_{1 i^{*}}\right|^{2}\right) \approx$ $\left(Q / /\left.h_{1 i^{*}}\right|^{2}\right)$ and $\min \left(P, Q /\left|h_{2 j^{*}}\right|^{2}\right) \approx\left(Q /\left|h_{2 j^{*}}\right|^{2}\right)$. In other words, the fixed interference power constraint becomes the dominant factor. By setting $L_{R}=L_{D}=1$ and $L_{R}=L_{D}=3$, we see that TAS/MRC outperforms TAS/GSC and TAS/GSC outperforms TAS/SC.

\section{CONCLUSIONS}

We took into account the cognitive DF relay network with TAS/GSC over Nakagami- $m$ fading. This framework is well suited for the reliability enhancement of the secondary network and interference alleviation of the primary network. We derived new statistical properties of the end-to-end SNR. Based on these, we derived closed-form expressions for the exact and asymptotic ergodic capacity with proportional and fixed interference constraints. Our results are valid for Nakagami- $m$ fading with arbitrary number of antennas. Our results demonstrate that: 1) under the proportional interference power constraint, the high SNR slope is independent of the interference power constraint, the number of antennas at the source, relay, and destination, as well as the primary network; and 2) under the fixed interference power constraint, a capacity ceiling exists.

\section{REFERENCES}

[1] A. Goldsmith, S. A. Jafar, I. Maric, and S. Srinivasa, "Breaking spectrum gridlock with cognitive radios: An information theoretic perspective," Proc. IEEE, vol. 97, no. 5, pp. 894-914, May 2009.

[2] C. Zhong, T. Ratnarajah, and K.-K. Wong, "Outage analysis of decodeand-forward cognitive dual-hop systems with the interference constraint in Nakagami- $m$ fading channels," IEEE Trans. Veh. Technol., vol. 60, no. 6, pp. 2875-2879, Jul. 2011.

[3] T. Q. Duong, P. L. Yeoh, V. N. Q. Bao, M. Elkashlan, and N. Yang, "Cognitive relay networks with multiple primary transceivers under spectrum-sharing," IEEE Signal Process. Lett., vol. 19, no. 11, pp. 741744, Nov. 2012.

[4] Y. Deng, M. Elkashlan, P. L. Yeoh, T. Duong, and R. K. Mallik, "Generalized selection combining in cognitive MIMO relay networks," in Proc. IEEE Int. Communications Conf. (ICC), Sydney, Australia, Jun. 2014, pp. 1-5.

[5] R. Zhang and Y.-C. Liang, "Exploiting multi-antennas for opportunistic spectrum sharing in cognitive radio networks," IEEE J. Sel. Topics Signal. Process., vol. 2, no. 1, pp. 88-102, Feb. 2008.

[6] V. Blagojevic and P. Ivanis, "Ergodic capacity for TAS/MRC spectrum sharing cognitive radio," IEEE Commun. Lett., vol. 16, no. 3, pp. 321323, Mar. 2012.

[7] Q. Wu, Y. Huang, J. Wang, and Y. Cheng, "Effective capacity of cognitive radio systems with GSC diversity under imperfect channel knowledge," IEEE Commun. Lett., vol. 16, no. 11, pp. 1792-1795, Nov. 2012.

[8] N. B. Mehta, S. Kashyap, and A. F. Molisch, "Antenna selection in LTE: from motivation to specification," IEEE Commun. Mag., vol. 50, no. 10, pp. 144-150, Oct. 2012.

[9] I. S. Gradshteyn and I. M. Ryzhik, Table of Integrals, Series and Products, 7th ed. San Diego, C.A.: Academic Press, 2007.

[10] E. Telatar, "Capacity of multi-antenna gaussian channels," Eur. Trans. Telecomm., vol. 10, no. 6, pp. 585-595, 1999. [Online]. Available: http://dx.doi.org/10.1002/ett.4460100604

[11] H. Shin and J. H. Lee, "Capacity of multiple-antenna fading channels: spatial fading correlation, double scattering, and keyhole," IEEE Trans. Inf. Theory, vol. 49, no. 10, pp. 2636-2647, Oct. 2003.

[12] S. S. Ikki and S. Aissa, "Multihop wireless relaying systems in the presence of cochannel interferences: Performance analysis and design optimization," IEEE Trans. Veh. Technol., vol. 61, no. 2, pp. 566-573, Feb. 2012.

[13] S. Jin, M. R. McKay, C. Zhong, and K.-K. Wong, "Ergodic capacity analysis of amplify-and-forward MIMO dual-hop systems," IEEE Trans. Inf. Theory, vol. 56, no. 5, pp. 2204-2224, May. 2010.

[14] E. Bjornson, P. Zetterberg, M. Bengtsson, and B. Ottersten, "Capacity limits and multiplexing gains of MIMO channels with transceiver impairments," IEEE Commun. Lett., vol. 17, no. 1, pp. 91-94, Jan. 2013. 